\title{
The study on soil moisture monitoring based on water-cloud model
}

\author{
Huang Zuo-wei ${ }^{1,2}$, chi xiang ${ }^{1}$, Xie Shi-xiong ${ }^{1}$ \\ ${ }^{1}$ School of Architecture and urban planning, Hunan University of Technology, Zhuzhou \\ 412000,China \\ 2 School of Geosciences and Information-Physics, Central South University, Changsha \\ 410083,China
}

Keywords: backscatter coefficient, water-cloud model, moisture monitoring

\begin{abstract}
Based on the use of optical image data, this paper uses NDMI to estimate the vegetation water content. It employs multi-polarization satellite-based radar data together with microwave scattering Water-Cloud Model eliminating the impact of the vegetation layer and isolating the contributions of vegetation scattering and absorption from the total backscattering coefficient, the result shows that the combination of $\mathrm{C}$-band $\mathrm{HH}$ polarization radar data with visible image performs well in the study of removing vegetation influence while retrieving soil water content in medium vegetated areas.
\end{abstract}

\section{Introduction}

With the fast development of urbanization,both industrial and residential domestic water consumption have increased significantly,causing the regional water table to decline each year,which has created water-use conflicts. The groundwater resource has a limited and non-renewable character. Soil moisture monitoring of microwave remote sensing has experienced 30-year history, and has been established many backscattering coefficient models such as small perturbation model, wo-scale model and the integral equation model and so on.In addition,there are experience and semi-empirical models.However,in the vegetated areas of arid and semi-arid oasis, the application of these retrieval models are confined by surface roughness and vegetation coverage.Higher vegetation coverage will cause lower estimation on soil moisture and the higher estimation on surface roughness which make the acquisition of soil moisture became complex. The key question to the study of soil moisture is how to eliminate the influence of surface roughness and separate the vegetation scattering and absorption from the soil moisture.

\section{Dada and Processing}

The multi-polarization data of Radarsat-2 in September 18,2010 is chosen in xinjiang district,and the polarization is $\mathrm{HH}$ and HV.The homochronous visible data is Landsat-5 TM data which includes mid-infrared and near-infrared band for calculating NDMI.The image of study area is shown Fig.1. 


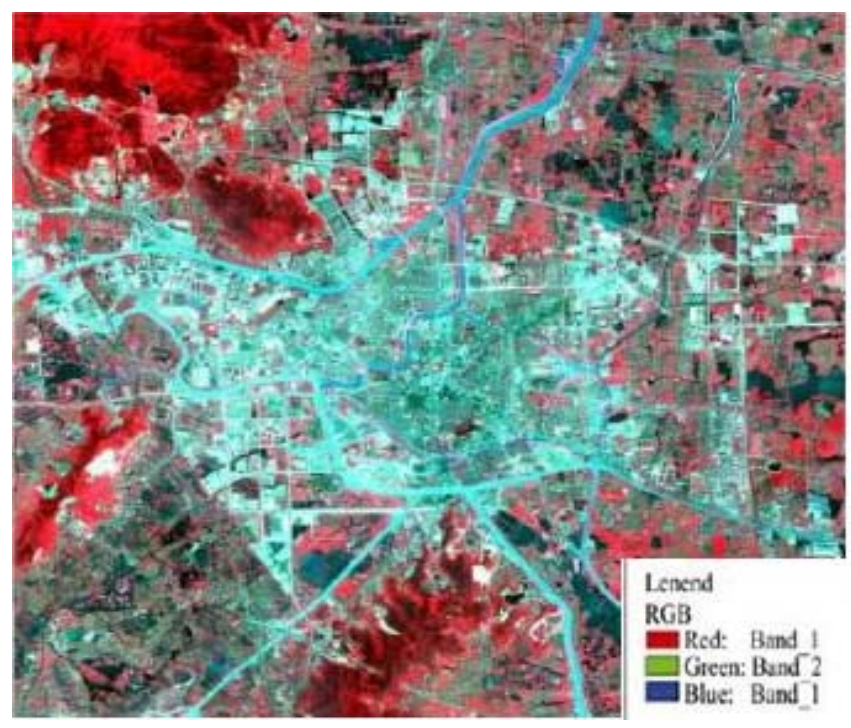

Fig.1 Polarization composite image of RADARSAT 2

Radarsat-2 satellite was successfully launched in December 14,2007 at space launch base in Kazakhstan's Baikonur,it can provide 11 kinds of beam patterns, the highest resolution is $3 \mathrm{~m}$, the maximum imaging width is $500 \mathrm{~km}$, and the maximum data rate is $445.4 \mathrm{Mb} / \mathrm{s}$, the range of incidence angle is $10^{\circ}-60^{\circ}$. The $\mathrm{C}$-Band can effectively extract soil moisture of $0-5 \mathrm{~cm}$ soil layer.

Landsat-5 TM data obtained in Sep 27,2008 is used as a reference map t choose the typical samples and combining with GPS positioning technology.Sampling points are selected regularly distribution as much as possible and comprehensive considering of the local soil,vegetation type and other factors. Sampling time is from sep 16,2008 to sep 26, 2008 and there are 30 located sampling points in this study.Set 5 sampling points as Plum-shape in the range of $30 \mathrm{~m}$ of each located points and profile mining each sample points and the profiles of each sample are $0-5 \mathrm{~cm}$. Borrow these soil samples and vegetation samples around of the soil sample dots back to laboratories, and dry them with the weighing method to obtain the soil avoirdupois moisture and vegetation water content.

Image data pre-processing mainly includes image registration,geometric correction,noise reduction and color enhancement and so on.As it is need to find out the relationship between normalized difference moisture index(NDMI)and vegetation water content(VWC), the atmospheric correction of TM data is absolutely necessary. The level of SAR image data obtained is SLC,so it should do noise reduction,radiometric calibration and geometric correction.

in order to keep the aboriginality,the image only did at-mospheric correction and geometric correction neglecting other pre-processing, which assure the objectivity of estimation.Because of the impacts of atmosphere on visible-near-infrared and thermal infrared are different, it is necessary to separately carry out atmospheric correction using $6 \mathrm{~S}$ model. While geometric correction was performed to do multinomial correction of TM image using ground control points.

SIGMA parameter correction is selected.Figure out the distance orientational incident angle of every pixels, then RADARSAT-2 satellite image data is changed to backscattering coefficient using the below calibration expression. The interrelated treatment in the processing of Radar-sat imaging causes a large number of spots(Speckle), which produce an obstacle on feature extraction,so the noise treatment is used to eliminate the impact.After several rounds of test analysis, $5 \times 5$ enhanced Lee filter to the original SAR image has the best filtering effect,which can eliminate the most spots.In this thesis,the study area is flat,so cubic polynomial is selected as the correction method.

\section{Method}

The vegetation water content(VWC)is extracted based on a choice of appropriate microwave scattering model.As it is definitely difficult to do large-scale area survey and extracting, which also definitely destroys vegetation, this paper uses Landsat-5 TM data to figure out normalized difference moisture index(NDMI)and find out the relationship between NDMI and VWC, then validates the 
vegetation water content.With that,multi-polarization satellite-based radar data together with microwave scattering water-cloud model are employed eliminating the impact of the vegetation layer on radar backscattering and finally retrieves the soil water content of study area.

\section{Extracting of characteristic parameters and vegetation water content estimation}

The vegetation water content is defined as the water content of plants per unit and per area.As a significant input parameter of Water-Cloud model,VWC performs very crucial effect on the retrieval of soil moisture in vegetated areas. Now, the study of the regional retrieval of VWC using visible image has been already mature,and the relativity established between spectrum index and VWC is high.So,this study uses visible remote sensing to retrieve and field survey data of samples to validate the VWC in

Table 1 Parameters input to MIMICS modelstudy area.In view of the spectrum characteristic of soil,vegetation and water between 0.8 to $1.7 \mu \mathrm{m}$, The spectrum bandwidths are respectively $25 \mathrm{~nm}$ and $20 \mathrm{~nm}$.According to the characteristic of vegetation that it has higher reflectivity in near-infrared band and lower reflectivity in mid-infrared band because of the absorbing effect of leave's water content,NDMI is applied to extract vegetation water content information. As the vegetation in study area are most cotton and other low salt secretion plants, according to the relationship between vegetation water content of field survey and NDMI.

$\mathrm{NDMI}=(\mathrm{NIR}-\mathrm{MIR}) /(\mathrm{NIR}+\mathrm{MIR}), \mathrm{VWC}=2.15 \mathrm{NDMI}+0.32$

There are rivers,lakes and reservoirs in study area which disturb the extracting of vegetation water content.So,the effects of these water bodies must be removed in the process of the retrieval work. This paper uses MNDWI index(Xu,2008) which is improved a little according as the regional circs to extract water body information,the equation is as follows:MNDWI=(1+0.5)(GREEN-MIR)/(GREEN+MIR)

After that,masking the original image and the water body effects are removed.Analysis and validation indicate that NDMI performs well in the retrieval of vegetation water content.

\section{Calculating of backscattering in vegetated areas}

The soil moisture estimation of initiative microwave remote sensing is mainly affected by vegetation coverage and surface roughness. The soil backscattering in vegetated area is composed of body scattering from vegetation,surface scattering from earth and the multiple scattering between vegetation layer and the surface. These factors are taken into account in the estimation of soil moisture content.As the hypsography in study area is flat,and mostly is covered by low plants, vegetation is considered seriously while the effect of surface roughness is not taken into account when calculating the soil backscattering coefficient. In the study of soil moisture estimation in vegetated area,it is necessary to use"Water-Cloud"model to remove the contri bution of vegetation in the backscattering.Parameters $\mathrm{A}$ and $\mathrm{B}$ are experience constants and they are changed by regional limit.A relatively mature method is applied to calibrate the parameters(Chen et al.,2007;Gaoetal.,2008).Firstly,Water-Clou $\mathrm{d}$ and MIMICS model are used to simulate the backscattering coefficient of vegetated area then obtain the surface direct scattering and the variational relationship between surface scattering and incident angle.Follow that,parameters of the dominate plants in study area is showed in Table 1.

Table 1 Parameters input to MIMICS model

\section{Result}

\begin{tabular}{|l|l|l|}
\hline Class & Parameters & Value \\
\hline \multirow{3}{*}{ Sensor } & Frequency/GHz & 5.4 \\
\cline { 2 - 3 } & Polarization mode & $\mathrm{HH} / \mathrm{HV}$ \\
\hline \multirow{3}{*}{ Land surface } & Water content & 0.17 \\
\cline { 2 - 3 } & Root mean square & 0.817 \\
\cline { 2 - 3 } & Correlative length/cm & 7.528 \\
\hline \multirow{5}{*}{ Leaves } & Water content & 0.39 \\
\cline { 2 - 3 } & Radius/cm & 2.6 \\
\cline { 2 - 3 } & Thickness/cm & 0.04 \\
\cline { 2 - 3 } & Height $/ \mathrm{m}$ & 0.9 \\
\hline Branch & Radius/cm & 0.3 \\
\cline { 2 - 3 } & Length/cm & 21 \\
\cline { 2 - 3 } & Density/m & 8.6 \\
\hline Climate & Temperature & 22 \\
\hline
\end{tabular}



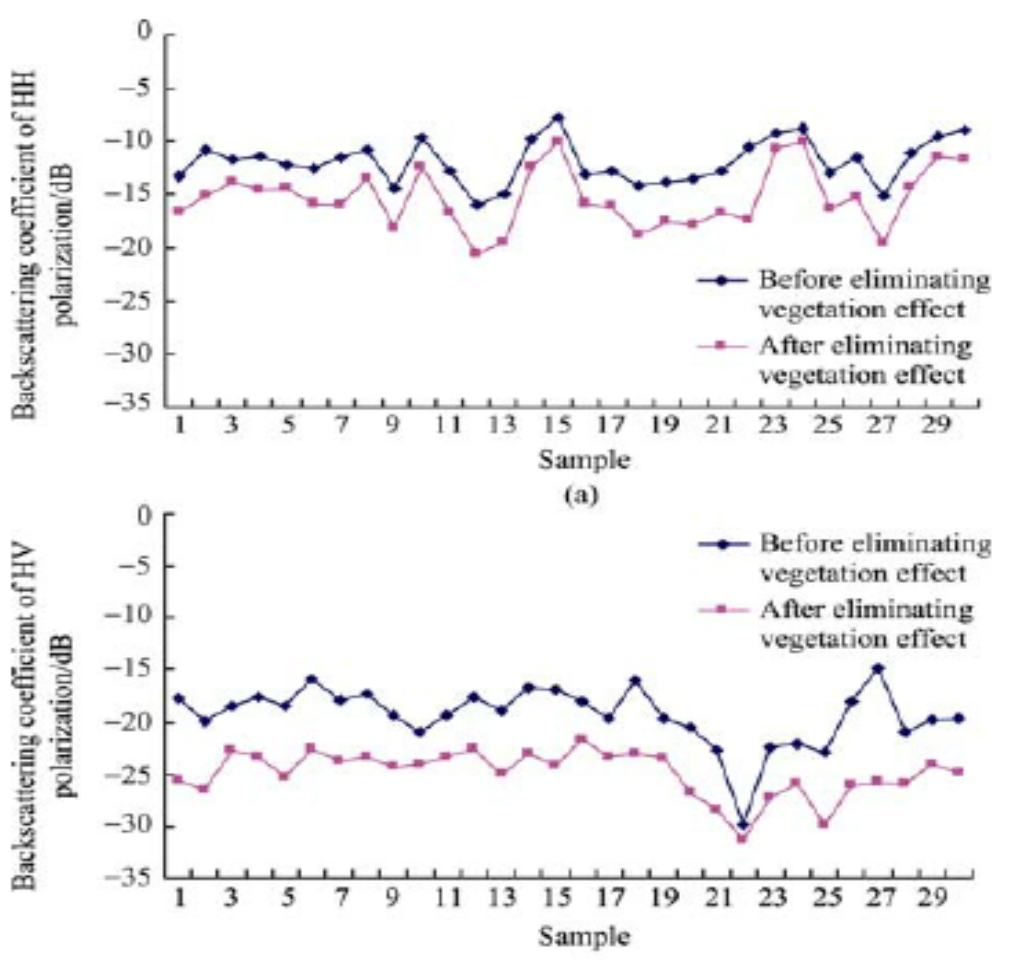

(b)

Fig. 2 relationship between $\mathrm{HH}, \mathrm{HV}$ polarization backscattering coefficient

(a)HH polarization;(b)HV polarization

we got 30 sample dots from field survey which include water content in the soil layer of $0-5 \mathrm{~cm}$ and the coordinates of these samples.Backscatteringcoefficient and water content of soil of the samples are showed. respectively analyze the relationship between $\mathrm{HH}, \mathrm{HV}$ polarization backscattering coefficient and water content of soil before and after removing vegetation influence and discuss the impact to backscattering coefficient of vegetation. Obtain the corresponding backscattering coefficient of the 30 samples in the image according as their coordinates, the relationship between before and after removing vegetation influence of $\mathrm{HH}, \mathrm{HV}$ polarization backscattering coefficient displayed in Fig.2.

It is showed that, $\mathrm{HH}, \mathrm{HV}$ polarization backscattering coefficient are becoming attenuation when separating the scattering and absorbing of vegetation using water-cloud model.The change of $\mathrm{HH}$ polarization data is thin,the reason is thatthe cropland of these samples is over the irrigation time and the evaporation is high, which results in lower water content of leaves and rhizome. Thus, the impact to scattering and absorbing of vegetation is tiny.HV polarization data is influenced by the scattering of plant canopy and branch. The study area is mostly covered by low crops and shrub.It is supposed that the vegetation is a orbicular scattering body covered with the earth's surface and ignoring its size,figure and the distributing character of the orientation, which results in a tiny change afterremoving the influence of vegetation. It illustrate the correlation coefficient of $\mathrm{HH}, \mathrm{HV}$ polarization backscattering coefficient and soil water content before eliminating vegetation effect are respectively 0.3769 and 0.1919 , while them rise to 0.5227 and 0.3277 with the use of Water-Cloud Model after eliminating vegetation effect. 


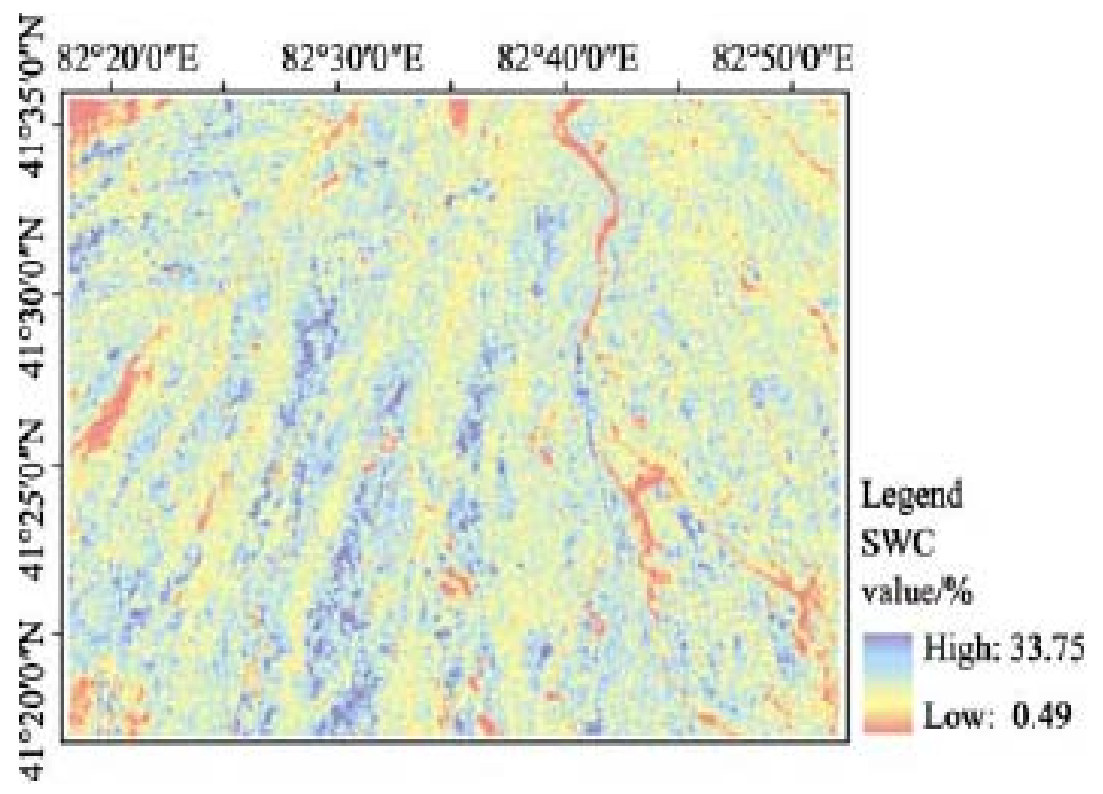

Fig.3 Soil water content map

Therefore, the relativity of soil backscattering coefficient andsoil water content is increasing while eliminating the influence of vegetation. Research indicated $\mathrm{HH}$ polarization is much more sensitive to soil water content,data in this paper validate that HH polarization combining with Water-Cloud Model performs well in the separation of vegetation's scattering and absorbing from the total backscattering of soil. Based on the above conclusion,establishing the connection model of soil water content and $\mathrm{HH}$ polarization backscattering coefficient, Use this method to retrieve the samples'soil water content, results is as Fig.3

\section{References}

[1] Gao S,Niu Z and Liu C Z.2008. The estimation of tropical plantation forest leaf area index based on Radarsat SAR data.Remote Sensing for Land\&resources, 78(4):35-38

[2] Jackson T J,Chen D and Cosh M.2004.Vegetation water content mapping using Landsat data derived normalized difference water index for corn and soybeans.Remote Sensing of Environment [3] Liao J J,Guo H D, Shao Y,LI X W and Veneziani N.2002.Method and model of surface feature detection in arid to Semi-arid Area Using SAR Interferometry.

[4] Li Z,Guo H D and Shi J C.2002.Measuring the change of soil moisture with vege tation cover integration passive and active microwave data.Journal of Remote Sensing,6(6):481-484

[5] Liu W X,Liu X L and Wang J.2008.Romote sensing retrieval of soil moisture using ENVISAT-ASAR and MODIS images in vegetated areas of Huanan.Agricultural Research in the Arid Areas,26(3):39-43

[6] Sun Z,Wang Q,Matsushita B,Fukushima T,Ouyang Z and Watanabe M. 2009.Development of a simple remote sensing evapotranspriation model(Sim-ReSET):Algorithm and model test.Journal of Hydrology,(376):476-485

[7] Teixeira A H C,Bastiaanssen W G M,Ahmad M D and Bos M G.2009. Reviewing SEBAL input parameters for assessing evapotranspiration and water productivity for the Low-Middle Sao Francisco

River Basin,Brazil.Part B:Application to the regional scale.Agricultural and ForestMeteorology, 149(3):477-490 